INPLASY

PROTOCOL

To cite: Rathod et al. The impact of timing of concurrent chemoradiation in limited stage SCLC undergoing platinum-based chemotherapy. Inplasy protocol 202180049. doi:

10.37766/inplasy2021.8.0049

Received: 12 August 2021

Published: 12 August 2021

Corresponding author:

Shrinivas Rathod

srathod@cancercare.mb.ca

Author Affiliation:

CancerCare Manitoba and

University of Manitoba.

Support: None.

Review Stage at time of this submission: Data analysis.

Conflicts of interest:

None declared.

\section{The impact of timing of concurrent chemoradiation in limited stage SCLC undergoing platinum-based chemotherapy}

\author{
Rathod, S1; Liang, Y2; Dubey, A3; Jeremic, B4.
}

Review question / Objective: Does the timing of concurrent chemoradiation in limited stage SCLC undergoing platinumbased chemotherapy affect outcomes.

Condition being studied: Limited stage small cell lung cancer. Eligibility criteria: Phase II and III design RCT evaluating the timing of radical RT (early vs late) in LS SCLC treated with platinum-based CHT and involving concurrent schedule of CRT will be potentially eligible for inclusion in this review.Initiation of RT with cycle 1 or 2 of $\mathrm{CHT}$ is considered as "early RT" and initiation of RT with later CHT is considered as "late RT". Phase II and III design RCT evaluating the timing of radical RT (early vs late) in LS SCLC treated with platinumbased CHT and involving concurrent schedule of CRT will potentially be eligible for inclusion in this review.

INPLASY registration number: This protocol was registered with the International Platform of Registered Systematic Review and Meta-Analysis Protocols (INPLASY) on 12 August 2021 and was last updated on 12 August 2021 (registration number INPLASY202180049).
Rationale: Small cell lung cancer (SCLC) occurs in around $15 \%$ of all lung cancer cases. 1 The ratio of cases of limited stage (LS) to extensive stage (ES) is usually described as $1: 2$, so that only about $5 \%$ of all lung cancer cases fall into a LS SCLC.2 It is, therefore, not with the great surprise that clinical research is this setting developed slowly with major findings rarely changing pattern of practice worldwide. In the past several decades, many 
approaches had been tested including radiation therapy (RT) alone, chemotherapy (CHT) alone and even, although extremely rarely, surgery alone. However, breakthrough seemingly came from the combination of RT and CHT. One of the clinical questions frequently asked after two meta-analyses findings that proved combination is better than CHT alone in the past was "optimal timing" of administration of RT and CHT, in other words, it meant when during the CHT course RT would start. Several studies attempted to give the answer to this question starting as early as almost 35 years ago with inconclusive results. Possible reasons for differing results would include heterogeneity of study populations (due to changing diagnostic approaches) and treatments administered. Regarding the latter issue, there was a number of RT (total dose, type of fractionation, dose per fraction) and CHT (drugs, doses, frequency) characteristics that varied over the time. In an attempt to solve the issue, several meta-analyses were performed with somewhat conflicting results: while some showed superiority for "early" administration of RT and CHT14, others showed no difference between two approaches15. It seemed that all of these meta-analyses suffered from inherent characteristics of the prospective randomized studies included in their analyses. As a consequence, no approach had been suggested as preferred one. In order to answer the same question, but also make eventual results more applicable in the clinic, we will embark on an approach of addressing this issue by focusing upon what we believed was characteristic of "standard" treatments, RT given using continuous course RT and platinum-etoposide $\mathrm{CHT}$ regimens. We will focus on the phase II and phase III studies that had asked the question of optimal timing of administration of concurrent RT and CHT (CCRT), intentionally excluding studies which used non-concurrent and non-continuous course RT as well as exclusively using non-platinum-etoposide CHT, since these are not considered "standard" treatments nowadays.
Condition being studied: Limited stage small cell lung cancer.

\section{METHODS}

Search strategy: We will systematically search electronic databases of Pubmed and Embase and restrict the search strategy to randomized controlled trials using the McMaster HIRU balanced therapy filter20 and studies published in the English language only. We will use additional search filters to exclude animal studies, studies involving the pediatric population and nonrelevant publication types such as comment or editorial or news or newspaper article or letters. Details of the search strategy are elaborated on in Appendix. The reference lists of relevant studies will be hand-searched to identify any potential additional studies. Deduplication of identified studies and management of bibliographic citations will be performed using commercially available reference management tool Endnote version 20 (https://endnote.com). Screening of potential studies and selection of eligible studies will be performed using Rayyan ai a free webbased intelligence systemic review platform (https://www.rayyan.ai). The initial title and abstract screening will be performed by two independent authors (SR and BJ). Any potential citation deemed eligible at the title and abstract screening by either reviewer will be included for the full-text review. The full-text articles will be assessed for inclusion based on the full inclusion/ exclusion criteria. Any disagreement will be resolved by consensus through mutual discussion and any disagreement will be resolved through third reviewer (AD) mediation.

Participant or population: Limited stage small cell lung cancer-Phase II and III design RCT evaluating the timing of radical RT (early vs late) in LS SCLC treated with platinum-based CHT and involving concurrent schedule of CRT will be potentially eligible for inclusion in this review. 
Intervention: Early concurrent chemoradiation (Radiation with cycle 1 or 2 of chemotherapy).

Comparator: Late concurrent chemoradiation (Radiation with beyond cycle 2 of chemotherapy).

Study designs to be included: Phase II and III design RCT.

Eligibility criteria: Phase II and III design RCT evaluating the timing of radical RT (early vs late) in LS SCLC treated with platinum-based $\mathrm{CHT}$ and involving concurrent schedule of CRT will be potentially eligible for inclusion in this review.Initiation of RT with cycle 1 or 2 of CHT is considered as "early RT" and initiation of RT with later CHT is considered as "late RT". Phase II and III design RCT evaluating the timing of radical RT (early vs late) in LS SCLC treated with platinumbased CHT and involving concurrent schedule of CRT will potentially be eligible for inclusion in this review.

Information sources: We will systematically search electronic databases of Pubmed and Embase.

Main outcome(s): This review will assess overall survival (OS) as the primary outcome measure.

Additional outcome(s): Progression free survival (PFS), complete response (CR) rate, any response rate (CR, partial response-PR, and stable disease-SD), 5year local control and 5-year brain control will be evaluated as secondary outcome measures. Acute and late toxicity (grade III and higher) will be assessed for safety outcome measures.

Data management: This systematic review and meta-analysis will be conducted in accordance with Cochrane handbook for systematic reviews of interventions. We will Grades of Recommendation, Assessment, Development, and Evaluation (GRADE) to assess quality of evidence. Preferred Reporting of Systematic Reviews and Meta-Analyses (PRISMA) guidelines will be used in the preparation of this article.19 The lead team will have assess to the raw data and would be shared upon request.

Quality assessment / Risk of bias analysis: We will assess the risk of bias using Cochrane risk of bias for RCT.

Strategy of data synthesis: We will extract demographic, treatment, and outcome data from the included studies. Demographic (author, publication year, no of participants, age), intervention (thoracic RT- total dose, schedule, dose per fraction, and RT volumes; CHT drug regimen and schedule), co-interventions including prophylactic cranial radiation $(\mathrm{PCI})$ use and dose, and duration of follow up) and outcomes (OS, PFS, CR rate, any response rate, 5-year local control rate, 5-year brain control rate, acute and late toxicity rates) data will be collected from the included publications. Two authors (SR and YL) will independently extract and pool the outcome data using the Cochrane methodology for metaanalysis. For the time to event outcomes, OS and PFS, the hazard ratio (HR) with $95 \%$ confidence interval $(\mathrm{Cl})$ and $p$-value will be recorded. If the HR was not reported in the publication, the authors of the included studies will be contacted for relevant information. If authors could not provide the data, time-to-event data (HR with 95\% $\mathrm{Cl})$ will be extracted from the survival curves (Kaplan-Meier curves were analyzed by Engauge Digitizer version 10.7) or with methods using $p$-value, number of patients randomized to each arm and number of events using the previously reported methods. The log $\mathrm{HR}$ and its variance will be pooled using inverse variance weighted average method (DerSimonian-Laird fixed-effects model) and expressed as a HR or odds ratio (OR), as appropriate, with respective $95 \% \mathrm{Cl}$ and p-value. Heterogeneity will be assessed by I^2 percentage that expresses the percentage of the variability in the effect sizes that is not caused by sampling error, and heterogeneity variance $\tau^{\wedge} 2$ that quantifies the variance of the true effect sizes underlying the data. Random-effects model will be selected if significant statistical heterogeneity $\left(12 I^{\wedge} 2>50 \%\right)$ is 
observed. Beg and Egger funnel plot method will be applied to find out any publication biases. Dichotomous outcomes as CR rate, any response rate, 5-year local control rate, 5-year brain control rate will be assessed using pooled risk ratio (RR). Uncommon events as grade III acute and late toxicity rates will be assessed OR. The statistical analysis will be performed (YL and SR) using the statistical software Revman version 5.4 .

Subgroup analysis: Based on CHT regimen and type of radiation administered subgroup analysis will be planned.

Sensitivity analysis: Appropriate sensitivity analysis will be planned based on available data.

Language: English only.

Country(ies) involved: Canada and Serbia.

Other relevant information: None

Keywords: small cell lung carcinoma, limited stage, radiation, platinum chemotherapy, timing, systematic review and meta-analysis.

Dissemination plans: Conference presentation; Publication in indexed journals.

Contributions of each author:

Author 1 - Shrinivas Rathod - Concept, literature search, data collection, data extraction, analysis, manuscript drafting and edits.

Email: srathod@cancercare.mb.ca

Author 2 - You Liang - data collection, data extraction, analysis, manuscript drafting and edits.

Email: you.liang@ryerson.ca

Author 3 - Arbind Dubey - literature search and manuscript edits.

Email: adubey@cancercare.mb.ca

Author 4 - Branislav Jeremic - Concept, literature search, data collection, data extraction, analysis, manuscript drafting and edits.

Email: nebareje@gmail.com 